\title{
Templated Oligosaccharide Synthesis: The Linker Effect on the Stereoselectivity of Glycosylation
}

\author{
Papapida Pornsuriyasak, Xiao G. Jia, Sophon Kaeothip, and Alexei V. Demchenko* \\ Department of Chemistry and Biochemistry, University of Missouri-St. Louis, One University \\ Boulevard, St. Louis, Missouri 63121, United States
}

\section{Abstract}

\begin{abstract}
A new method for intramolecular oligosaccharide synthesis that is conceptually related to the general molecular clamp approach is introduced. Exceptional $\alpha$-selectivity has been achieved in a majority of applications. Unlike other related concepts, this approach is based on the bisphenol A template, which allows one to connect multiple building blocks to perform templated oligosaccharide synthesis with complete stereoselectivity. This principle was demonstrated by the synthesis of an $a, a$-linked trisaccharide.
\end{abstract}

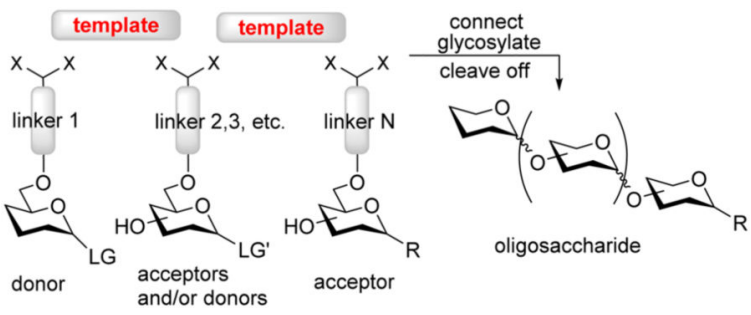

\begin{abstract}
The past decade has witnessed a renaissance in oligosaccharide synthesis. ${ }^{1}$ Recent discoveries in the synthetic field have been driven by the increasing understanding of the crucial roles that oligosaccharides play in many fundamental biological processes. ${ }^{2}$ Access to certain classes of complex carbohydrates from natural sources is feasible, although the quantity and purity of the isolated material often do not meet the current demand. Chemical synthesis represents the major mode for obtaining relatively large quantities of pure material. However, the limited synthetic capability demands a search for new efficient technologies that would enable both expeditious assembly and reliable control of the stereoselectivity at each coupling step. This is of particular relevance to the synthesis of 1,2-cis among other challenging glycosidic linkages. ${ }^{3}$
\end{abstract}

\footnotetext{
*Corresponding Author, demchenkoa@umsl.edu. Author Contributions

The manuscript was written through contributions of all authors./All authors have given approval to the final version of the manuscript.

Supporting Information

The Supporting Information is available free of charge on the ACS Publications website at DOI: 10.1021/acs.orglett.6b01102.

Experimental details and characterization data (PDF)

The authors declare no competing financial interest.
} 
The issue of controlling the stereoselectivity of glycosylations has been approached in a variety of modes. ${ }^{4,5}$ For instance, intramolecular approaches occupy an important niche among other methods available. Many attractive intramolecular glycosylation methods have emerged during the past two decades.$^{6-9}$ The underlying concept is that two glycosylation components, the glycosyl donor and acceptor, are tethered together using a cleavable linker. The purpose of this tethering is to achieve efficient facial selectivity due to steric or geometric constraints or forces that may help to control the stereoselectivity of the glycosylation. These techniques are classified into three major subgroups: intramolecular aglycone delivery (IAD), leaving-group-based methods, and the "molecular clamp" concept (approaches A-C in Figure 1).

The molecular clamp concept ${ }^{10}$ links the glycosyl donor and acceptor away from the reaction sites. Realistically, this approach allows one only to glycosylate a hydroxyl adjacent to the tether on the acceptor site. An example of this approach is the use of a rigid xylylene tether that can be conveniently removed concomitantly with debenzylation. ${ }^{11}$ Overall, molecular-clamp-directed glycosylation is a three-step process: (1) formation of the intermolecular tether between the glycosyl donor and a free hydroxyl of the glycosyl acceptor; (2) intramolecular structurally restricted glycosylation; and (3) removal of the tether. The investment in the additional steps is often compensated by the ability to access challenging glycosidic bonds that are otherwise hard to accomplish by direct protocols.

Herein we introduce a new concept, templated oligosaccharide synthesis, in which the tethering can be achieved away from the reactive sites. In contrast to the other concepts, the templated approach enables glycosylation of different hydroxyls, not only those adjacent to the tether. It also provides exceptional $a$-selectivity and allows multiple building blocks to be connected for oligosaccharide synthesis. The general outline of a templated synthesis is shown in Scheme 1. If the synthesis of a disaccharide is targeted (Scheme 1A), a glycosyl donor equipped with linker 1 is connected to a glycosyl acceptor bearing linker 2 via a template. The resulting donor-acceptor tethered pair is then subjected to glycosylation, and the disaccharide is cleaved off the template. In cases where the synthesis of an oligosaccharide is attempted, a series of building blocks equipped with various linkers are connected via a template in a sequential manner (Scheme 1B). The tethered donor-acceptor network is then subjected to glycosylation. Finally, the resulting oligosaccharide is cleaved off the template.

To execute this concept, we considered the following. To be practical for synthetic applications, the linkers and the template itself should be easy to install and remove. Also, by variation of the length of the linker it should be possible to control the stereoselectivity of the subsequent glycosylation. For instance, if the linker connecting the acceptor is longer than that of the donor, this should facilitate the delivery of the nucleophile from the bottom (a) face of the donor. Having learned from previous work by Fairbanks and co-workers ${ }^{12,13}$ and Warriner ${ }^{14}$ as well as our own experimentation (see the Supporting Information (SI)) with flexible peptide-based templates, we anticipated that the rigidity of the template would critically affect the stereoselection. With these considerations in mind, we prepared various donor-acceptor conjugates based on bisphenol A (BPA) as the rigid template (see the SI for the synthesis). 
The first series of conjugates $\mathbf{1}, \mathbf{3}$, and $\mathbf{5}$ were designed to deliver the glycosyl acceptor from the bottom face. In this case the donor linker was shorter than that of the acceptor (succinoyl (Suc) vs glutaryl (Glt), respectively). The key results of this study are summarized in Table 1. When a perbenzylated donor tethered with a 4-OH acceptor (1) was activated with MeOTf, a mild activator for thioglycosides, ${ }^{15}$ followed by the ester bond cleavage under Zemplen conditions $(\mathrm{NaOMe})$ and standard acetylation $\left(\mathrm{Ac}_{2} \mathrm{O} /\right.$ pyridine $)$, disaccharide $\mathbf{2}$ was isolated in $81 \%$ yield with complete $\alpha$-selectivity (entry 1 ); no traces of the $\beta$-linked diastereomer were detected. It should be noted that many other promoters of the activation of thioglycosides, including dimethyl(thiomethyl)sulfonium triflate (DMTST) ${ }^{16}$ and NIS/ $\mathrm{TfOH}$, gave shorter reaction times, but the yields were lower because of competing hydrolysis. When MeOTf-promoted activation was applied to glycosylation of the tethered 3-OH acceptor 3, disaccharide 4 was obtained in 73\% yield with complete $a$-selectivity (entry 2). Glycosylation of the tethered 6-OH acceptor 5 provided disaccharide 6 in 63\% yield. Again, the preference was for formation of the $a$-linked product, although the presence of the other diastereomer was also evident $(\alpha / \beta=9.2 / 1$; entry 3$)$. It is possible that the compromised stereoselectivity in this case is related to the fact that the primary 6-OH is more flexible and can be reached from both the bottom and top faces of the activated donor (oxacarbenium ion intermediate). ${ }^{17}$

Having controlled the $\alpha$-stereoselectivity with the longer acceptor linker, we were curious to investigate the stereoselectivity of glycosylations with spacers of equal length. For this purpose we obtained a tempate with succinoyl linkers for the attachment of both the glycosyl donor and acceptor (7). Complete $a$-stereoselectivity was still maintained in glycosylations of acceptors with secondary hydroxyls, and disaccharide 2 was obtained in $76 \%$ yield (entry 4). With good reaction yields and excellent $a$-stereoselectivity achieved in most templatemediated glycosylations, we also investigated a template wherein the glycosyl acceptor would be expected to be delivered from the top $(\beta$ ) face. For this purpose, the glycosyl donor was attached to the donor counterpart via a longer linker (Glt) than that of the glycosyl acceptor (Suc). In the case of compound $\mathbf{8}$, however, practically no selectivity was achieved. Entry 5 clearly shows a typical outcome, which implied that the synthesis of $\beta$ linked derivatives could not be accomplished using this approach.

We postulated that enhanced rigidity might provide a more stringent acceptor delivery mode and hence help to improve the stereoselectivity for primary glycosyl acceptors. To investigate this, we prepared the tethered compounds $\mathbf{9}$ and $\mathbf{1 0}$, which were subjected to the same reaction conditions. The tethered 4-OH acceptor $\mathbf{9}$ gave disaccharide $\mathbf{2}$ in an impressive $90 \%$ yield with complete $\alpha$-selectivity (entry 6). Even more importantly, MeOTfpromoted synthesis of the $(1 \rightarrow 6)$-linked disaccharide $\mathbf{6}$ from the tethered donor-acceptor 10 also resulted in an excellent yield of $89 \%$ with complete $a$-selectivity (entry 7).

Having achieved excellent yields and complete stereocontrol in all of the syntheses of $\alpha$ linked disaccharides, we were curious to see whether essentially the same approach could be used for the synthesis of $\beta$-linked disaccharides. For this purpose we prepared a benzoylated glycosyl donor that was tethered with a 3-OH acceptor (11). MeOTf-promoted glycosylation was rather sluggish $(40 \mathrm{~h})$, perhaps because of the disarmed nature of the perbenzoylated donor used herein. ${ }^{18}$ Nevertheless, the reaction progressed smoothly, and disaccharide $\mathbf{1 2}$ 
was obtained in $84 \%$ yield with complete $\beta$-stereoselectivity (entry 8 ). The rate of this coupling could be significantly enhanced in the presence of NIS/TfOH (10 min), but the isolated yield of disaccharide $\mathbf{1 2}$ was reduced. Interestingly, when the respective benzylated donor was glycosylated in $\mathrm{MeCN}$, a reaction solvent that is known to enhance $\beta$ selectivity, ${ }^{19}$ still only the $\alpha$-linked disaccharide was obtained. This result implies that the effect of the intramolecular tethering on the stereoselectivity of glycosylation is significantly stronger than that of the reaction solvent.

We next explored the possibility of extending the one-step glycosylation reactions to multistep processes for the synthesis of longer oligosaccharide sequences. For this purpose, donor-linker-template conjugate $\mathbf{1 3}$ was reacted with trimellitic anhydride in the presence of EDC and DMAP. The resulting intermediate $\mathbf{1 4}$ was obtained in $98 \%$ yield. It was reacted with building block 15 (see the SI for the synthesis) to afford tethered donor-acceptor pair $\mathbf{1 6}$ in 95\% yield (the combined yield for $\mathbf{1 6}$ and its meta-linked regioisomer). In contrast to the previously executed reactions with simple linkers, intermediate $\mathbf{1 6}$ could be used for further template elongation via the carboxyl group. Indeed, BPA-conjugated glycosyl acceptor 17 could be linked to compound 16 using a DCC/DMAP-mediated coupling reaction to obtain the templated conjugate $\mathbf{1 8}$ in $82 \%$ yield. The synthesis of compound $\mathbf{1 8}$ clearly demonstrated that all of the additional steps required for the installation of the template tethering could be achieved effectively and in very high yield.

The templated compound $\mathbf{1 8}$ was designed in such a way that the unit on the left-hand side could act as the glycosyl donor only. The unit on the right-hand side could act as the glycosyl acceptor only, whereas the central building block could act either as the glycosyl acceptor or the donor upon demand. With a number of possibilities available, so far we have explored only the one shown in Scheme 2. First, activation of the $S$-ethyl leaving group in compound 18 was achieved with MeOTf, and the glycosylation of the middle building block took place with concomitant removal of the $p$-methoxybenzyl (PMB) group. The latter could be also removed as a separate step prior to glycosylation, but in our hands the one-pot procedure provided a slightly higher yield. It should be noted that essentially the same principle for direct glycosylation of hydroxyls masked with temporary PMB protection created a basis for the development of the reverse orthogonal approach. ${ }^{20}$ Second, the $\sigma$ allylphenyl leaving group ${ }^{21,22}$ was activated with NIS/TfOH, and again the PMB group of the acceptor was removed during the glycosylation step. The resultant tethered trisaccharide was released from the template by reaction with $\mathrm{NaOMe}$ in $\mathrm{MeOH}$ to afford compound 19 in $58 \%$ overall yield with complete stereoselectivity for both glycosidic linkages.

In conclusion, we have developed a new concept for intramolecular glycosylation and oligosaccharide synthesis. Complete stereoselectivity has been achieved in a majority of applications. In particular, the rigid bisphenol A template and phthaloyl linkers permit highly stereoselective glycosidic bond formation for both primary and secondary glycosyl acceptors. Efficient intramolecular glycosylation with glycosyl donors equipped with a nonparticipating benzyl group at C-2 led to the exclusive formation $\alpha$-linked disaccharides. $a$-Selectivity can be lower in the case of primary glycosyl acceptors and flexible linkers. We also demonstrated that $\beta$-linked glycosides can be efficiently formed with the aid of a participatory effect of the neighboring ester group. Moreover, we demonstrated the 
possibility of extending the template to the synthesis of a trisaccharide, which was also obtained with complete $a$-stereoselectivity for both glycosylation steps. Further development of this methodology and its application to oligo- and polysaccharide synthesis in currently underway in our laboratory.

\section{Supplementary Material}

Refer to Web version on PubMed Central for supplementary material.

\section{ACKNOWLEDGMENTS}

This work was supported by the National Institute of General Medical Sciences (GM111835). Dr. Winter and Mr. Kramer (UM-St. Louis) are thanked for HRMS determinations.

\section{REFERENCES}

1. Smoot JT, Demchenko AV. Adv. Carbohydr. Chem. Biochem. 2009; 62:161. [PubMed: 19501706]

2. Varki, A.; Cummings, RD.; Esko, JD.; Freeze, HH.; Bertozzi, CR.; Stanley, P.; Hart, GW.; Etzler, ME. Essentials of Glycobiology. 2nd ed.. CSH Laboratory Press; Cold Spring Harbor, NY: 2009.

3. Nigudkar SS, Demchenko AV. Chem. Sci. 2015; 6:2687. [PubMed: 26078847]

4. Mydock LK, Demchenko AV. Org. Biomol. Chem. 2010; 8:497. [PubMed: 20090962]

5. Crich D. Acc. Chem. Res. 2010; 43:1144. [PubMed: 20496888]

6. Jung KH, Muller M, Schmidt RR. Chem. Rev. 2000; 100:4423. [PubMed: 11749353]

7. Madsen, J.; Bols, M. Carbohydrates in Chemistry and Biology. Ernst, B.; Hart, GW.; Sinay, P., editors. Vol. 1. Wiley-VCH; Weinheim, Germany: 2000. p. 449

8. Ziegler, T. Handbook of Chemical Glycosylation. Demchenko, AV., editor. Wiley-VCH; Weinheim, Germany: 2008. p. 469

9. Ishiwata A, Lee YJ, Ito Y. Org. Biomol. Chem. 2010; 8:3596. [PubMed: 20585666]

10. Ziegler T, Lau R. Tetrahedron Lett. 1995; 36:1417.

11. Muller M, Huchel U, Geyer A, Schmidt RR. J. Org. Chem. 1999; 64:6190.

12. Tennant-Eyles RJ, Davis BG, Fairbanks AJ. Chem. Commun. 1999:1037.

13. Tennant-Eyles RJ, Davis BG, Fairbanks AJ. Tetrahedron: Asymmetry. 2000; 11:231.

14. Burt J, Dean T, Warriner S. Chem. Commun. 2004:454.

15. Lonn H. J. Carbohydr. Chem. 1987; 6:301.

16. Ravenscroft M, Roberts RMG, Tillett JG. J. Chem. Soc., Perkin Trans. 1982; 2:1569.

17. Whitfield DM. Adv. Carbohydr. Chem. Biochem. 2009; 62:83. [PubMed: 19501705]

18. Fraser-Reid B, Wu Z, Udodong UE, Ottosson H. J. Org. Chem. 1990; 55:6068.

19. De Meo C, Farris M, Ginder N, Gulley B, Priyadarshani U, Woods M. Eur. J. Org. Chem. 2008; 2008:3673.

20. Fujikawa K, Vijaya Ganesh N, Tan YH, Stine KJ, Demchenko AV. Chem. Commun. 2011; 47:10602.

21. Luo S-Y, Tripathi A, Zulueta MML, Hung S-C. Carbohydr. Res. 2012; 352:197. [PubMed: 22370177]

22. Premathilake HD, Demchenko AV. Beilstein J. Org. Chem. 2012; 8:597. [PubMed: 22563357] 

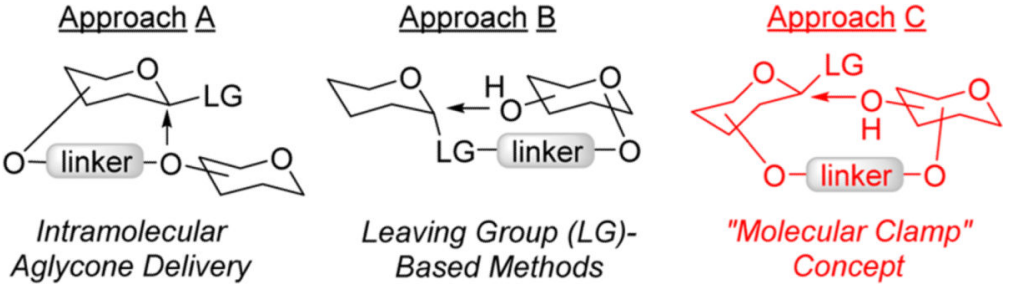

Figure 1.

Three general concepts for the intramolecular glycosylation reaction. 

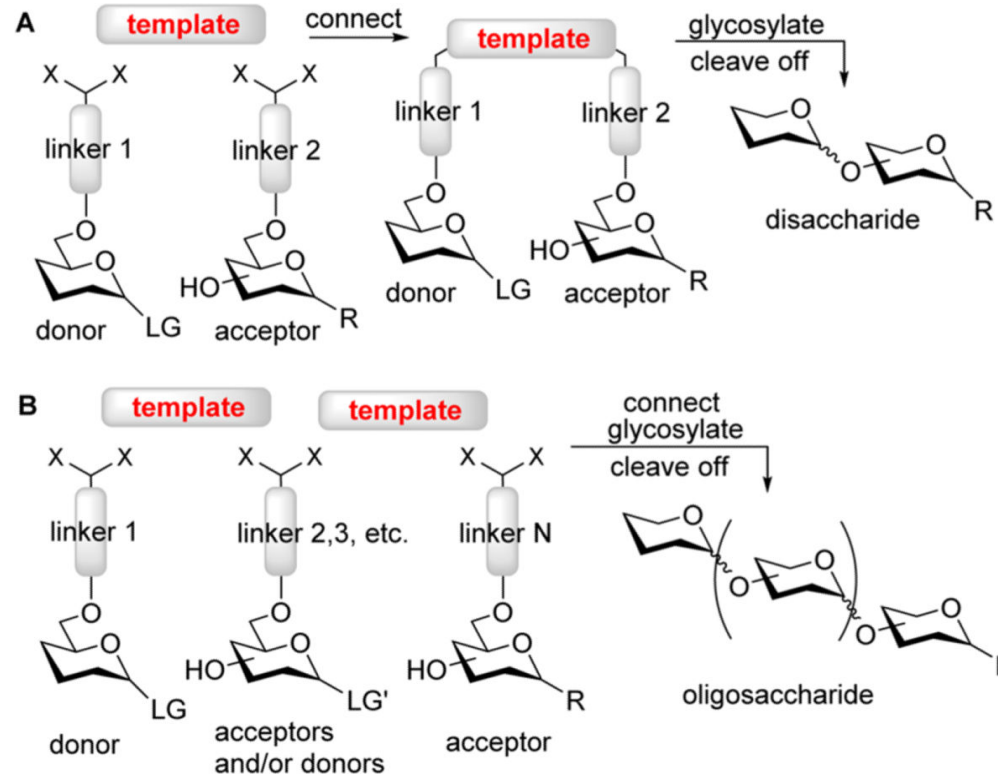

connect

glycosylate

cleave off

linker

o and/or donors

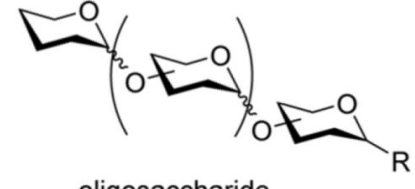

oligosaccharide

Scheme 1.

Outline of the Concept of Templated Glycosylation (A) and Oligosaccharide Synthesis (B) 


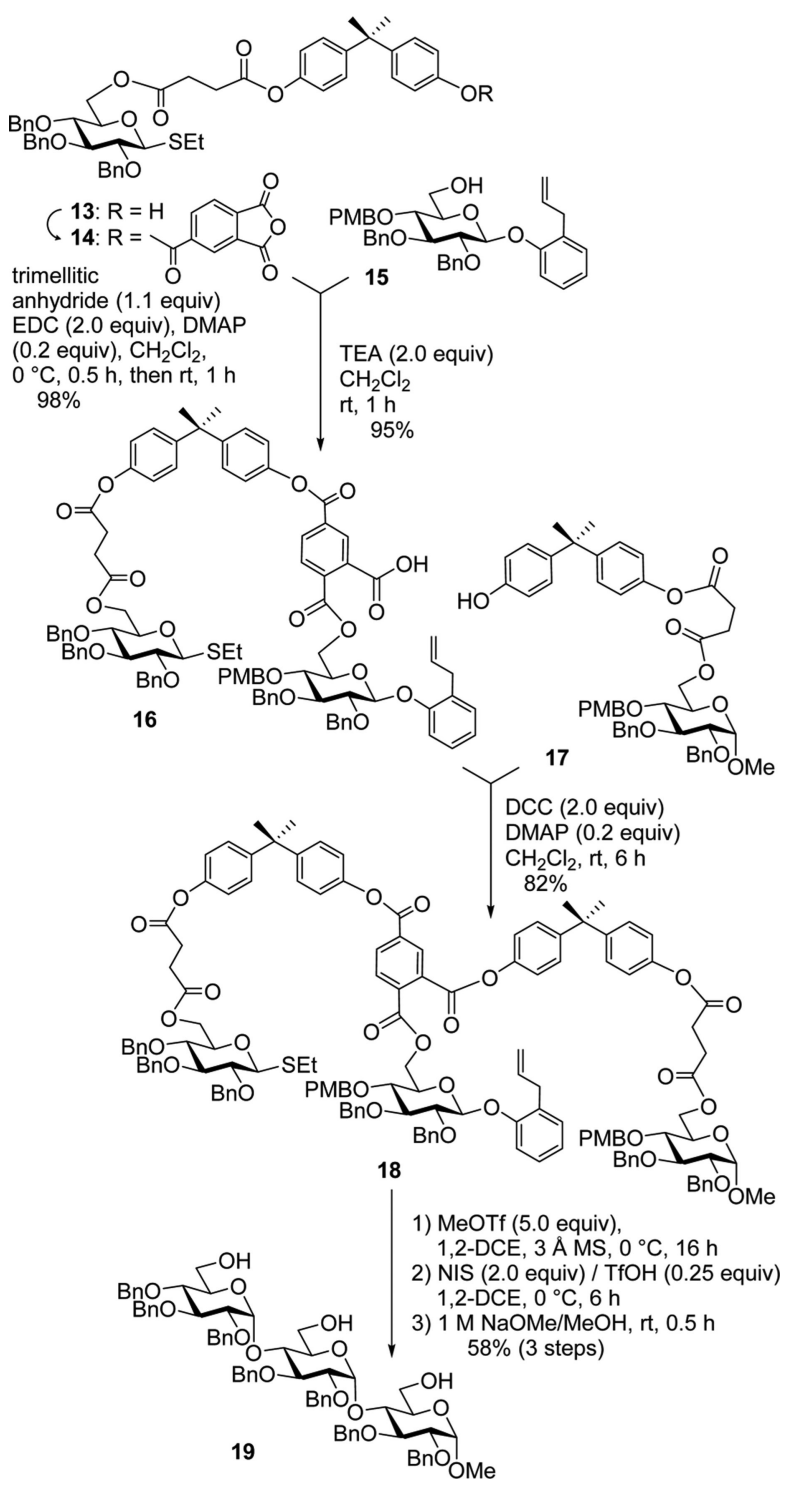

Scheme 2.

Templated Synthesis of $a, a$-Linked Trisaccharide 19 


\section{Table 1}

Linker Effect on the Stereoselectivity of Glycosylation

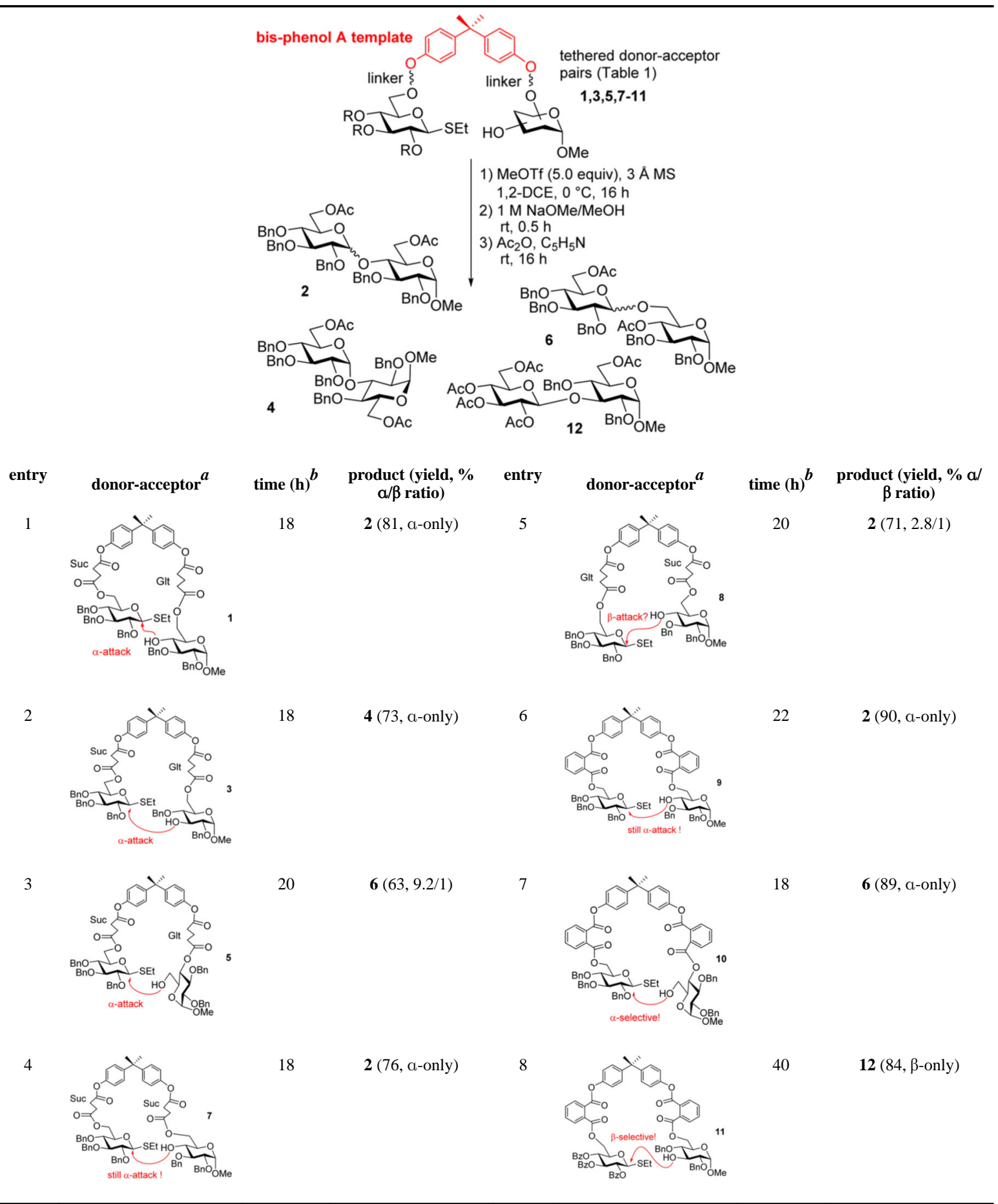

${ }^{a}$ Suc $=$ succinoyl; Glt $=$ glutaryl .

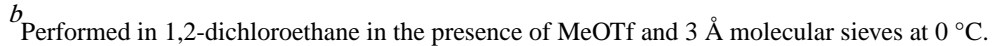

Org Lett. Author manuscript; available in PMC 2016 June 17. 